\title{
Signature of metastable electrons in highly charged ion surface interactions
}

\author{
J. Ducrée*, J. Mrogenda, E. Reckels, M. Rüther, A. Heinen, Ch. Vitt, M. Venier, \\ J. Leuker, and H.J. Andrä \\ Institut für Kernphysik, Westfälische Wilhelms-Universität Münster, Wilhelm-Klemm-Str. 9, \\ D-48149 Münster, Germany
}

(October 29, 2018)

\begin{abstract}
We present autoionization spectra of metastable $\mathrm{Ar}^{8+}$ and $\mathrm{C}^{4+}, \mathrm{N}^{5+}, \mathrm{O}^{6+}$ and $\mathrm{Ne}^{8+}$ scattering off an $\mathrm{Al}(111)$ surface with incident energies down to $5 \mathrm{eV}$. The unprecedented quality of the experimental data permits the observation of a unique in the structures originating from the metastable projectiles compared to corresponding ground state configurations. Analyzing the peak positions for different projectile species and velocities we demonstrate that the peak must be ascribed to an above-surface transition under participation of the metastable state.
\end{abstract}

32.80.Dz, 79.20.Rf, 34.50.Dy, 34.60.+z

Typeset using REVTEX

\footnotetext{
*Author to whom correspondence should be addressed. Electronic address: ducree@unimuenster.de
} 
The interactions of highly charged ions (HCIs) with surfaces have been studied extensively in the past, refer to [1] for a recent review. Several research groups have collected a tremendous amount of experimental data on Auger emission [2 [4], X-ray emission [5,6] and energy gains of reflected projectiles [7]. Also theoretical analysis has reached a remarkable level of sophistication [8,9]. Nevertheless, authors still argue about crucial issues of the interaction mechanism since a lot of the interpretations deduced from the experiment depend on certain model assumptions.

As a HCI approaches a metal surface it is neutralized via resonant charge transfer from the target valence band (VB) into its outer Rydberg states, see Fig. 1. Due to the image attraction, the interaction time of the incoming projectile in front of the surface possesses a principal lower limit of the order of $10^{-14} \mathrm{~s}$. Close above the first bulk layer atomic orbitals are energetically shifted and strongly distorted due to the bulk interaction and a theoretical description becomes intricate.

Authors have concluded from atomic structure calculations neglecting the bulk perturbations that a stepwise intra-atomic autoionization cascade proceeds too slow in order to carry all Rydberg state electrons into inner shells in front of the surface. Indeed, striking experimental evidence has been provided indicating that the overwhelming fraction of the inner-shell processes, in particular $K L L$ emission, takes place within the bulk region [9 11].

In this domain, the interactions between HCIs and the metal electron gas are fundamentally different. As the neutralized HCI reaches the first bulk layer it experiences the screening of the high density target electron gas and outer levels merge into the VB. Only electrons on the innermost projectile orbitals have a chance to survive bulk penetration. The HCI now acts as a point charge attracting a VB charge cloud to screen the positive core charge.

Since the image charge components and thus the potential barrier [12] which builds up between the HCI and the surface vanishes within the bulk the charge cloud electrons can directly replenish inner projectile levels via so called $L C V$ transitions [8, 15], see also Fig. 11. Compared with the slow intra-atomic cascade above the surface, this filling mechanism and 
succeeding Coster-Kronig regrouping [13] of the $L$-shell population proceed very rapidly and higher initial sublevel populations evolve before an inner shell transition takes place.

Auger transition energies increase significantly when inner-shell populations shield the core in a way that the statistically preferred shell configurations at the time of $K L L$ decay can be reconstructed via the most prominent spectral peak positions. Various efforts have quite successfully been conducted to develop an appropriate interaction model from these experimental clues combined with theoretical work [11,13.

In this paper we address the central issue of above-surface contributions in a new manner. In contrast to previous efforts in this field which partially rely on rather complex model assumptions concerning the evolution of the filling cascade [9,14 or secondary electron transport through the bulk region [10], the discovery of a new spectral structure allows us to only make use of simple, commonly accepted concepts.

\section{EXPERIMENTAL SPECTRA}

The HCIs are extracted by a fixed voltage of $-20 \mathrm{kV}$ from an ECR ion source floated on a selectable potential $U_{\mathrm{S}}$. After the ions are $q / m$ separated, a lens system focuses and decelerates the beam onto the grounded target in a UHV chamber $\left(p \simeq 5 \times 10^{-12} \mathrm{hPa}\right)$. An ion spectrometer, mounted close behind the removable target, measures the kinetic ion energy distribution the full width of which at half maximum never exceeded $2 \mathrm{eV}$ per charge. The beam energy $E_{\text {kin }}$ can freely be set by varying $U_{\mathrm{S}}$. The beam axis intersects the target surface at a variable angle $\Theta$. Electrons are detected perpendicular to the beam axis at an angle of $\Psi=90^{\circ}-\Theta$ with respect to the surface by an electrostatic $150^{\circ}$ spherical sector analyzer. The $\mathrm{Al}(111)$ surface has been prepared by successive cycles of sputtering and annealing and controlled by Auger electron spectroscopy.

In Fig. 2 we display two spectra of $\mathrm{Ar}^{8+}$ and $\mathrm{Ar}^{9+}$ projectiles impinging with $E_{\text {kin }}=$ $12.5 \mathrm{eV}$ per charge and $\Theta=5^{\circ}$ on an $\mathrm{Al}(111)$ surface. All spectra in this paper are normalized to the area below the portrayed peak region. The comparison with the $\mathrm{Ar}^{9+}$ spectrum 
yields a distinct, yet unseen peak in the well resolved $\mathrm{Ar}^{8+}$ spectrum on the upper fringe of the $L M M$ region which overlaps the $L M N$ region. Furthermore, a notable fraction of the $(3 p \mapsto 2 p, 3 p \uparrow)$ intensity in the dominant $211-\mathrm{eV}$ peak shifts to the $(3 s \mapsto 2 p, 3 \ell \uparrow)$ region $(\ell \in\{s, p\})$ at lower energies in the $\mathrm{Ar}^{8+}$ spectrum as a consequence of the additional $3 s$ electron. For higher perpendicular incident velocities at $\Theta=45^{\circ}$ the $247-\mathrm{eV}$ peak vanishes. Other aspects of these $\mathrm{Ar}^{q+}$ spectra have been outlined in [15].

In Fig. 3 we recorded a series of spectra of HCIs in metastable $1 s 2 s$ and hydrogenlike $1 s$ ground state configurations interacting with an $\operatorname{Al}(111)$ surface at $\Theta=5^{\circ}$ with $E_{\text {kin }}=$ $14.5 \mathrm{eV}$ per charge. On the analogy of the $\mathrm{Ar}^{8+}$ spectrum in Fig. 2, the additional peak (marked by vertical lines in the plots) within the $1 s 2 s$ spectra is superposed on the highenergy tail of the $K L M$ region. It is most prominent for $\mathrm{C}^{4+}$ and widens along the row towards $\mathrm{Ne}^{8+}$. For $\mathrm{C}^{4+}$ we added a spectrum measured at $E_{\text {kin }}=5 \mathrm{eV}$ and $\Theta=5^{\circ}$ on $\mathrm{Si}(100)$ to demonstrate that the height and sharpness of the additional peak can be enhanced by reducing the incident velocity. In other measurements on $\operatorname{Si}(100)$ we discovered nearly identical $K L V_{W}$ regions as on $\mathrm{Al}(111)$.

The greatest intensity of the spectra in Fig. 3 originates from $K L L$ transitions. The two sharp peaks ( $\mathrm{a}$ and b) on the low-energy side have previously [0,9] been assigned to $(2 s \mapsto 1 s, 2 s \uparrow)$ and $(2 s \mapsto 1 s, 2 p \uparrow)$ transitions out of low $L$-shell occupations. The upper part of the spectrum is partially composed of a $(2 p \mapsto 1 s, 2 p \uparrow)$ region (c) featuring a less pronounced peak on the high-energy side. With increasing effective core charge towards $\mathrm{Ne}^{8+}$ a well separated $K L M$ peak (d) emerges.

Fig. 3 unveils further striking discrepancies in the overall $1 s 2 s$ and $1 s$ peak structures. For the first time, we identify a systematic expansion of the $(2 s \mapsto 1 s, 2 s \uparrow)$ peak (a) to the low-energy side for metastable projectiles which is most pronounced for $\mathrm{Ne}^{8+}$. In a tentative explanation, we ascribe this remarkable shift to the close HCI-surface encounter region where the projectile core is still incompletely screened by the target electron gas while the stripping of outer Rydberg levels has already set in. Within the isotropic bulk environment, level shifts only depend on the spatially constant screening of valence band 
electrons and sharp peak profiles like the 211-eV peak in Fig. 2 occur. We postpone the discussion of this major effect to a future publication. Additionally, well defined subpeaks in the $(2 p \mapsto 1 s, 2 p \uparrow)$ (c) and a $K L M$ region (d) can be resolved. Complying with the previous peak assignment, regions (b) and (c) exhibit less intensity with respect to region (a) in the spectra of metastable projectiles.

\section{INTERPRETATION OF THE PEAK POSITION}

For the spectra of metastable configurations, the peculiar position of the additional peak on the high-energy tail of the $K L M$ spectra implies that apart from the $2 s$ electron which jumps into the $1 s$ vacancy an energy level situated far above the $M$-shell has to supply the emitted electron. In an attempt to find a consistent explanation for all HCI species we found that the peak position can most accurately be reproduced by putting the emitted electron in a projectile energy level which is resonant to the target work function $W$. The evaluation of the transition energy has been performed by the Cowan code [16 selecting $1 s 2 s n s^{x} n p^{y}$ as initial and $1 s^{2} n s^{x} n p^{y-1}$ as final configurations. The initial configuration is made neutral by choosing $x+y=q-2$ where $q$ denotes the nuclear projectile charge. $n$ represents the principal quantum number the $p$-subshell binding energy of which comes closest to $W$.

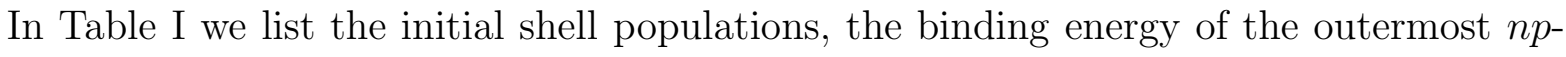
subshell, which should be compared to the Al work function of $4.25 \mathrm{eV}$, and the experimental and calculated peak energies. For each HCI species, including $\mathrm{Ar}^{8+}$ for which we replaced the core configuration $1 s 2 s$ by $2 p^{5} 3 s$, we attain surprisingly good agreement with the experimental values despite the simple evaluation method. In the following we denote these transitions by $K L V_{W}$ and for $\mathrm{Ar}^{8+}$ by $L M V_{W}$ and summarize the $n$-shells which are nearly resonant to $W$ by $V_{W}$. We now have to tackle the problem where this unique transition type takes place. The exclusive occurrence of the $K L V_{W}$ peak at low perpendicular incident velocities and its weak intensity suggest that it might proceed during the limited interaction

time in front of the bulk. Further evidence for this hypothesis comes from the scrutiny of 
the effective potentials seen by an active electron in front of and underneath the surface in the oncoming paragraphs.

The right hand side of Fig. 11 schematically depicts the deformation of the projectile potential close to the surface. In the classical overbarrier model [17] the charge transfer is described via one-electron Coulomb and image potentials. A narrow channel of typically a few eV width [12] classically permits a resonant charge flow between the HCI and the metal VB. The upper boundary of the channel is given by the highest occupied VB states, i.e. the work function $W$. The resonant capture is believed to constitute the most efficient above-surface neutralization mechanism.

For metastable projectiles, the resulting atomic electron configuration complies with the Cowan code simulation in Tab. [ and represents the ideal system to generate the $L M V_{W}$ and $K L V_{W}$ peaks in Figs. 2 and 3. The sharpness of the $K L V_{W}$ peak for metastable $\mathrm{C}^{4+}$, $\mathrm{N}^{5+}$ and the $L M V_{W}$ peak of $\mathrm{Ar}^{8+}$ suggests that captured electrons stay parked in the $V_{W}$ levels during the above-surface interaction phase which would be in contradiction to the mere cascade model [14. We assume that this behavior results from the dynamic interplay of resonant capture and loss and the elevation of projectile $V_{W}$ levels by image shifts. It is important to note these shifts vanish in commonly employed models [12] for the neutralized projectiles in Tab. 四. For the case of ionic projectiles, the continuum boundary limits the upward shifts of resonantly populated levels to less than $W$, i.e. a few eV only. Since Auger transition rates between two subshells critically depend on the energetic vicinity of the participating levels, high $K L V_{W}$ peak intensities cannot be expected despite the massive $V_{W}$ level occupation.

Within the bulk, the screening of the target electron gas lets the atomic potential merge into $V_{0}$ at large distances from the nucleus. $L C V$ processes and collisional side-feeding combined with Coster-Kronig processes quickly fill the atomic $L$-shell while pushing outer levels successively above $V_{0}$ (cf. Fig. 1). The binding energy of the $2 s$ level grows by several $10 \mathrm{eV}$ with increasing $L$ population. A below-surface transition involving the $(2 s \mapsto 1 s)$ part should therefore reflect the range of the $2 s$ binding energies convoluted with the VB 
electron density. This is in obvious contradiction to the small $K L V_{W}$ peak width.

Furthermore, at least one additional peak arising from $\left(2 p \mapsto 1 s, V_{W} \uparrow\right)$ transitions should appear because spectral lines on the high-energy side of the $K L L$ region representing $(2 p \mapsto 1 s, 2 p)$ transitions give evidence for a partially occupied $2 p$ level below the surface. In view of the fact that the spectra of metastable projectiles display a similar shape as those detected for their ground state counterparts like the $\mathrm{Ar}^{8+}$ and $\mathrm{Ar}^{9+}$ spectra above the 211eV peak in Fig. 2, we can additionally conclude that appreciable inner-shell populations do not occur as the ion descends towards the surface. We remark that the prominence of the $K L V_{W}$ peak in $\mathrm{C}^{4+}$ with respect to $\mathrm{N}^{5+}, \mathrm{O}^{6+}$ and $\mathrm{Ne}^{8+}$ might be rooted in their low binding energies of the metastable level possibly favoring high transition rates. Such a tendency has been found for $L C V$ transitions [8] the participating electron levels of which are separated in a similar way. At present, a more precise quantitative treatment for $K L V_{W}$ and $L M V_{W}$ processes is still missing.

With the preceding experimental evidence combined with our argumentation we supplied convincing proof that the distinct $K L V_{W}$ peak exclusively occurring in the spectra of metastable projectiles is generated before bulk penetration. Provided that a sufficient statistical quality of the spectra is available, the $K L V_{W}$ intensity contains valuable information on the interaction mechanisms and duration of the above-surface phase. Due to its low transition rate and the limited interaction time in front of the surface it sensitively reacts to changes in the perpendicular projectile velocity. The discovery of the $K L V_{W}$ peak therefore sets a novel benchmark for the scrutiny of previously proposed above-surface interaction models and opens an exciting perspective for future research on HCI-surface collisions. In particular for the very active field of HCI-insulator scattering, the $K L V_{W}$ and $L M V_{W}$ peak profiles may be exploited to experimentally study resonant charge transfer, atomic level shifts, projectile trajectories and energy deposition near the surface in a new manner.

This work was sponsored by the German Bundesministerium für Bildung, Wissenschaft, Forschung und Technologie under Contract No. 13N6776/4. We are also grateful for support from the Ministerium für Wissenschaft und Forschung des Landes NRW. One of us (JJD) 
appreciates fruitful discussions with U. Thumm and R. Díez Muiño. 


\section{REFERENCES}

[1] A. Arnau, F. Aumayr, P. M. Echenique, M. Grether, W. Heiland, J. Limburg, R. Morgenstern, P. Roncin, S. Schippers, R. Schuch, N. Stolterfoht, P. Varga, T. J. M. Zouros, and HP. Winter, Surface Science Reports 27, 113 (1997).

[2] F. W. Meyer, S. H. Overbury, C. D. Havener, P. A. Zeijlmans van Emmichoven, and D. M. Zehner, Phys. Rev. Lett. 67, 723 (1991).

[3] M. Grether, A. Arnau, R. Köhrbrück, A. Spieler, and N. Stolterfoht, Nucl. Instrum. Methods Phys. Res. B 115, 157 (1996).

[4] J. Thomaschewski, J. Bleck-Neuhaus, M. Grether, A. Spieler, D. Niemann, and N. Stolterfoht, Nucl. Instrum. Methods Phys Res. B 125, 163 (1997).

[5] H. J. Andrä, A. Simionovici, T. Lamy, A. Brenac, G. Lamboley, S. Andriamonje, J. J. Bonnet, A. Fleury, M. Bonnefoy, M. Chassevent, and A. Pesnelle, Z. Phys. D 21, suppl., 135 (1991).

[6] J.-P. Briand, B. d'Etat Ban, D. Schneider, M. A. Briere, V. Decaux, J. W. McDonald, and S. Bardin, Phys. Rev. A 53, 2194 (1996).

[7] H. Winter, J. Phys: Condens. Matter 8, 10149 (1996).

[8] R. Díez Muiño, N. Stolterfoht, A. Arnau, A. Salin, and P. M. Echenique, Phys. Rev. Lett. 76, 4636 (1996).

[9] J. Limburg, S. Schippers, I. Hughes, R. Hoekstra, R. Morgenstern, S. Hustedt, N. Hatke, and W. Heiland, Nucl. Instrum. Methods Phys. Res. B 98, 436 (1995).

[10] R. Köhrbrück, M. Grether, A. Spieler, N. Stolterfoht, R. Page, A. Saal, and J. BleckNeuhaus, Phys. Rev. A 50, 1429 (1994).

[11] M. Grether, A. Spieler, R. Köhrbrück, and N. Stolterfoht, Phys. Rev. A 52, 426 (1995).

[12] J. Ducrée, F. Casali, and U. Thumm, Phys. Rev. A 57, 338 (1998). 
[13] S. Schippers, J. Limburg, J. Das, R. Hoekstra, and R. Morgenstern, Phys. Rev. A 50, 540 (1994).

[14] J. Das and R. Morgenstern, Physical Review A 47, R755 (1993).

[15] J. Ducrée, J. Mrogenda, E. Reckels, M. Rüther, A. Heinen, Ch. Vitt, M. Venier, J. Leuker, R. Díez Muiño, and H. J. Andrä, Phys. Rev. A 57, 1925 (1998).

[16] R. D. Cowan, The Theory of Atomic Structure and Spectra (University of California Press, Berkeley, 1981).

[17] J. Burgdörfer, P. Lerner, and F. W. Meyer, Phys. Rev. A 44, 5674 (1991). 


\section{FIGURES}

FIG. 1. Sketch of the interaction scenario. Above the surface valence band electrons are resonantly transferred across the potential barrier into HCI Rydberg levels which accumulate large populations due to the slow intra-atomic cascade. The energy of the resonant Rydberg levels which we globally denote by $V_{W}$ remains constant at the target work function $W$ while quantum numbers may change. For metastable projectiles the $2 s$ electron can participate in a so called $K L V_{W}$ transition creating a peak which cannot occur during below surface emission. An analogous argumentation holds for the $L M V_{W}$ peak in $\mathrm{Ar}^{8+}$ spectra.

FIG. 2. Spectra of $\mathrm{Ar}^{9+}$ and metastable $\mathrm{Ar}^{8+}$ impinging with $E_{\mathrm{kin}}=12.5 q \mathrm{eV}$ at incident angles $\Theta=5^{\circ}$ and $45^{\circ}$ on $\mathrm{Al}(111)$, featuring an as yet undiscovered peak above the $L M M$ region for $\mathrm{Ar}^{8+}$ under the lowest perpendicular incident energy. At the $L M V_{W}$ peak, the absolute count rate amounts to 23000 electrons per $1 \mathrm{eV}$ channel and the statistical error is well below $1 \%$. We ascribe this peak to $L M V_{W}$ processes, cf. Fig. 1.

FIG. 3. Spectral series of metastable $(1 s 2 s)$ HCIs scattering off an $\mathrm{Al}(111)$ surface at $\Theta=5^{\circ}$

$E_{\text {kin }}$ and very low perpendicular incident velocities at $E_{\text {kin }}=14.5 q \mathrm{eV}$ and for $\mathrm{C}^{4+}$ also at $5 \mathrm{eV}$ on $\operatorname{Si}(100)$ with $W_{\mathrm{Si}}=4.6 \mathrm{eV}$. a, b, c and d label the $(2 s \mapsto 1 s, 2 s \uparrow),(2 s \mapsto 1 s, 2 p \uparrow),(2 p \mapsto 1 s, 2 p \uparrow)$ and $K L M$ regions which are well separated in the $\mathrm{Ne}^{9+}$ spectrum, respectively. The comparison with the corresponding $1 s$ configuration spectra reveals an additional $K L V_{W}$ peak (cf. Fig. 1) above the $K L M$ region (marked by a vertical line) which is most pronounced for $\mathrm{C}^{4+}$. At a typical count rate of 96000 electrons per 1-eV channel at the $\mathrm{C}^{4+} K L V_{W}$ maximum the experimental error falls far below $1 \%$. 


\section{TABLES}

\begin{tabular}{|c|c|c|c|c|}
\hline \hline \hline $\mathrm{HCI}$ & configuration & $V_{W}[\mathrm{eV}]$ & calc. peak energy $[\mathrm{eV}]$ & expt. peak energy $[\mathrm{eV}]$ \\
\hline $\mathrm{C}^{4+}$ & $1 s 2 s 4 s^{2} 4 p^{2}$ & 4.20 & 294.08 & $294 \pm 1$ \\
\hline $\mathrm{N}^{5+}$ & $1 s 2 s 4 s^{2} 4 p^{3}$ & 5.25 & 413.26 & $415 \pm 2$ \\
\hline $\mathrm{O}^{6+}$ & $1 s 2 s 5 s^{2} 5 p^{4}$ & 4.30 & 556.55 & $554 \pm 2$ \\
\hline $\mathrm{Ne}^{8+}$ & $1 s 255 s^{2} 5 p^{6}$ & 5.96 & 898.30 & $893 \pm 10$ \\
\hline $\mathrm{Ar}^{8+}$ & $2 p^{5} 3 s 6 s^{2} 6 p^{6}$ & 4.62 & 245.28 & $247 \pm 1$ \\
\hline \hline
\end{tabular}

TABLE I. $K L V_{W}$ and $L M V_{W}$ peak positions as determined by Cowan code simulation and

experiment. Also listed are the initial configurations entering the Cowan code input file and the binding energies of their outermost orbital which approximately equal the $\mathrm{Al}$ work function of $W=4.25 \mathrm{eV}$. 


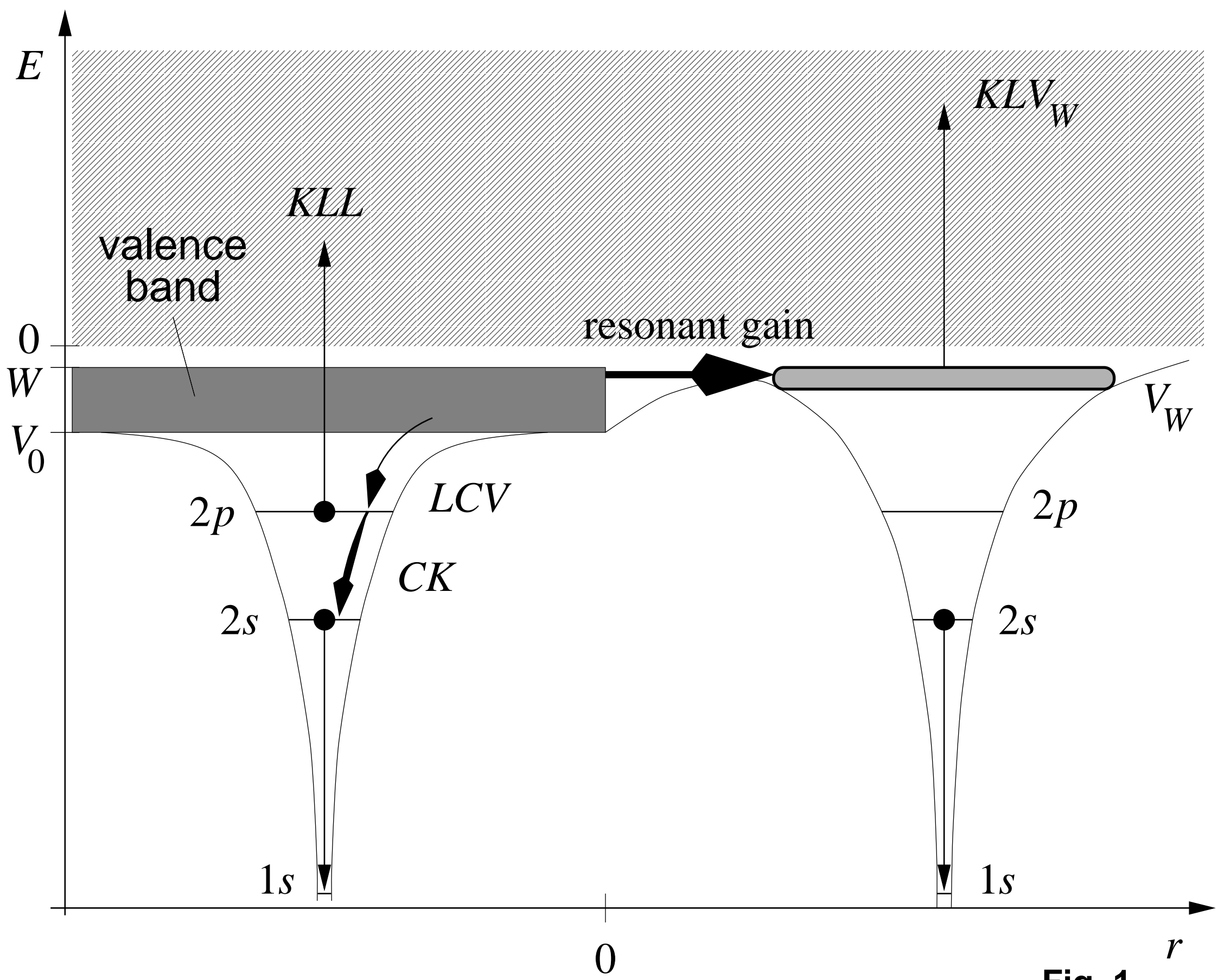

Fig. 1 


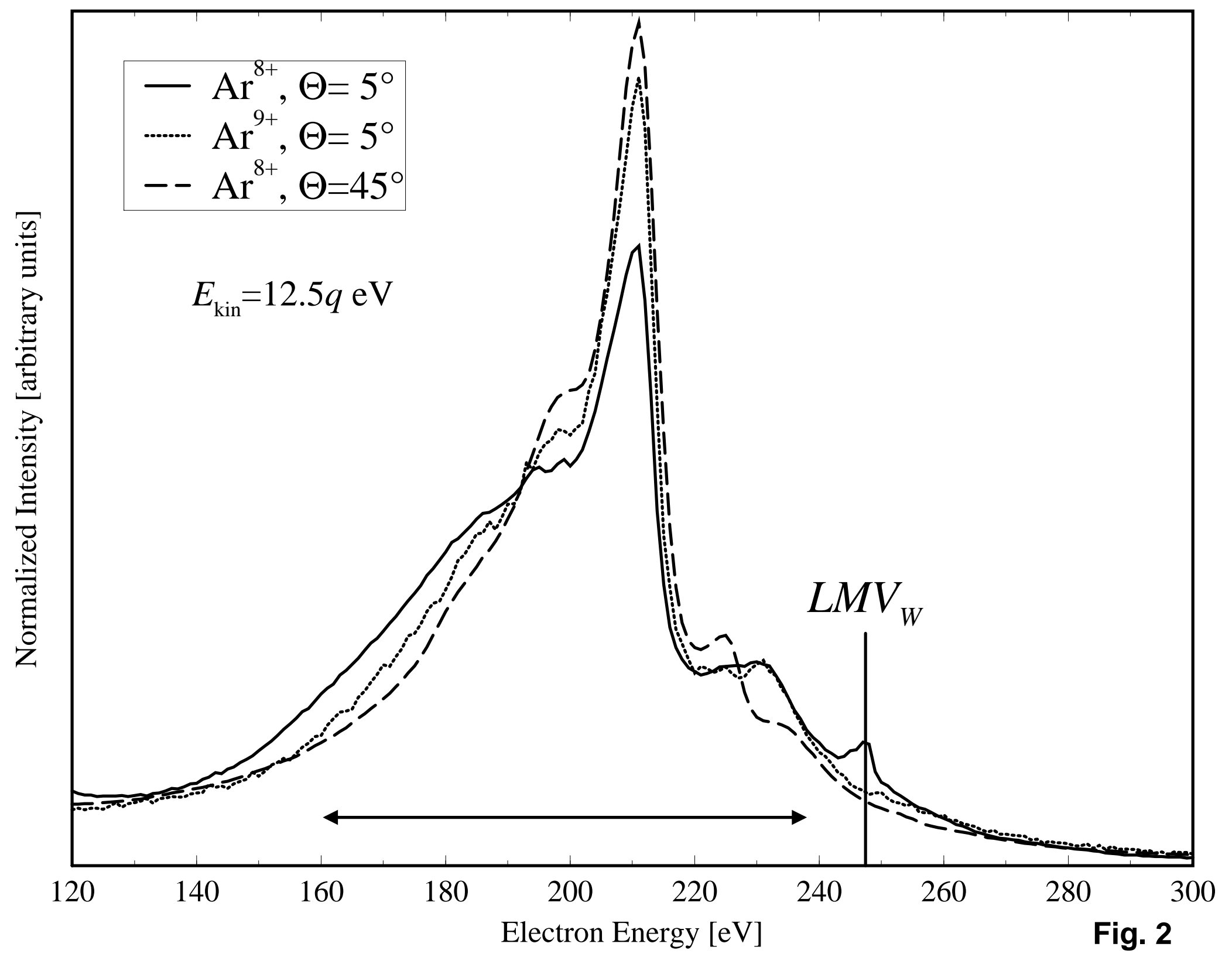




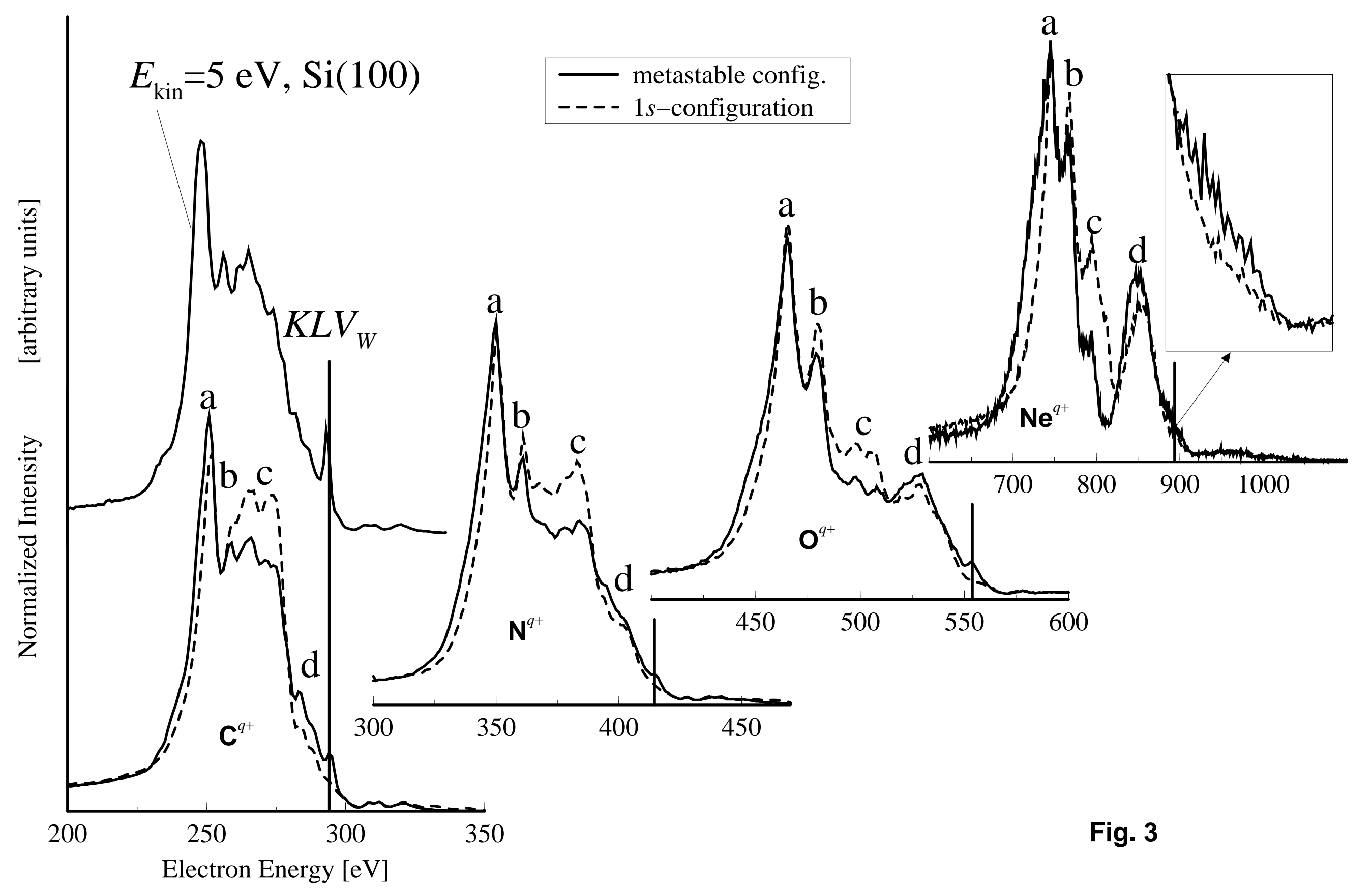

